\title{
Insights into the Impact of Transporter Proteins on the Uptake and Transport of Cerium Oxide Nanoparticles by Soybean (Glycine max. (L.) Merr.)
}

\author{
Xingmao Ma* \\ Zachry Department of Civil and Environmental Engineering, Texas A\&M University, \\ TAMU 3136, College Station, TX 77843-3136, USA \\ xma@civil.tamu.edu
}

\begin{abstract}
Broad application and disposal of engineered nanoparticles (ENPs) could lead to their accumulatin in plants and potentially affect food safety. Understanding the mechanisms for plant root uptake of ENPs is therefore critical. Cerium oxide nanoparticles $\left(\mathrm{CeO}_{2} \mathrm{NPs}\right)$ are used in a wide range of commercial products. Even though it is generally accepted that $\mathrm{CeO}_{2} \mathrm{NPs}$ are taken up by plant roots both as intact $\mathrm{CeO}_{2} \mathrm{NPs}$ and dissoluted $\mathrm{Ce}^{3+}$ ions, the moleuclar mechaisms for $\mathrm{CeO}_{2} \mathrm{NPs}$ internalization remain unknown. In this study, different ion channel blockers $\left(\mathrm{Gd}^{3+}, \mathrm{La}^{3+}\right)$ or protein synthesis and metabolic inhibitors (2,4-dinitrophenol, cycloheximide) were applied to asertain the molecular mechanisms for possible active transport of $\mathrm{CeO}_{2} \mathrm{NPs}$ and their dissolved ions into soybean roots (Glycine max. (L.) Merr.). The results suggest that active transporters such as calcium permeable transporters may be involved in the internalization of $\mathrm{CeO}_{2} \mathrm{NPs}$ and $\mathrm{Ce}^{3+}$.
\end{abstract}

Keywords: $\mathrm{CeO}_{2}$ nanoparticles; Ce ion, Calcium-permeable channels; Channel blockers, Soybean

\section{Introduction}

Engineered nanoparticles have been incorporated into agrochemicals (e.g., nano-pesticide, nano-fertilizers, nanosensors), cosmetics, textile, biomarkers, chemical and biological sensors, and medicine [1-4]. ENPs are now frequently detected in irrigation water and agricultural soils as a result of many intentional (e.g. agrochemicals) and/or unintentional releases (e.g. malfunction of wastewater treatment facilities) [5, 6].

Cerium oxide nanoparticles $\left(\mathrm{CeO}_{2} \mathrm{NPs}\right)$ have been extensively applied over the past years, particularly as a fuel additive [7, 8]. The main pathways for $\mathrm{CeO}_{2} \mathrm{NPs}$ to enter into agricultural lands are through deposition of vehicle exhausts and municipal runoff into the top soil [7]. Due to the potential phytotoxicity and accumulation of $\mathrm{CeO}_{2} \mathrm{NPs}_{\text {in agricultural }}$ products and the subsequent human health risks, it is imperative to understand the mechanisms for their uptake by plants [7, 9].

Even though $\mathrm{CeO}_{2} \mathrm{NPs}$ are generally deemed as stable in the environment, greater dissolution has been reported on root surface or in the rhizosphere, resulting in an additional pathway for Ce element to enter into plant tissues [10]. Root exudates were found to increase the solubility of $\mathrm{CeO}_{2} \mathrm{NPs}$ and mediate their accumulation in plant tissues [9]. The bioaccumulation of $\mathrm{CeO}_{2} \mathrm{NPs}$ in plant roots and subsequent transport to shoots may involve several complex pathways [5]. Passive diffusion through plant cell walls and membranes has been shown to be an important pathway for ENPs internalization [11]. Trimpathi et al. (2017) reviewed a massive body of literature and concluded that active-transport including signaling from calcium channels and the regulation of plasma membranes are also likely important for ENPs uptake [5]. The possibility of active transport of ENPs through different ion and protein channels into plant roots was also proposed by other researchers [9]. However, no conclusive evidence has been reported up to now to substantiate the assumption that ENPs are actively transported into plant roots through regulated ion channels. Schymura et al. 2017 used radiolabeling to investigate the uptake mechanism of $\mathrm{CeO}_{2} \mathrm{NPs}$ into plants, but their result was also inconclusive [7].

Ion and protein channels embedded in root membranes play significant roles in regulating the entrance of heavy metal ions into root cell cytoplasm [12,13]. Considering the dissolution of many metallic ENPs in plant rhizosphere, it is likely that the ion and protein channels will play some roles in ENPs internalization. The goal of this study was to shed new light in the roles of ion channels in the uptake of $\mathrm{CeO}_{2} \mathrm{NPs}$ and the dissolved Ce ions by plant roots. Application of calcium- 
permeable channel blockers or metabolic inhibitors (pharmaceuticals) is a well-established technique to understand the regulatory mechanisms of heavy metal uptake and transport in plants [12, 13]. However, this technique has not been explored to investigate the uptake mechanisms for ENPs and their associated ions. Soybean (Glycine max. (L.) Merr.) is used as a model plant because it is the fifth most produced crop worldwide, providing $30 \%$ vegetable oil, $77 \%$ natural nitrogen fixation and the annual demand for soybean products increases by $2.2 \%$ globally [14].

\section{Materials and Methods}

\subsection{Materials}

Lanthanum chloride ( $\geq 99.9 \%)$ and gadolinium (III) chloride hexahydrate ( $\geq 99.9 \%)$ were purchased from Acros Organics (Pittsburgh, PA) and Alfa Aesar (Ward Hill, MA, USA) respectively. 2,4-dinitrophenol (DNP, $\geq 98 \%$ ) was obtained from Ultra Scientific (Kingstown, RI, USA). Cycloheximide (CHX) and cerium (III) chloride heptahydrate $\left(\mathrm{CeCl}_{3} \cdot 7 \mathrm{H}_{2} \mathrm{O}, 99.9 \%\right)$ were purchased from Acros Organics (Pittsburgh, PA). The dispersion of polyvinylpyrrolidone (PVP) coated $\mathrm{CeO}_{2} \mathrm{NPs}$ (20\% by weight) was obtained from the USA Research Nanomaterials, Inc, (Houston, Texas). The nanoparticles are mostly spherical and have an average size of $10 \mathrm{~nm}$. The measured zeta potential was $51.57 \mathrm{mV}$ in a $500 \mathrm{mg} / \mathrm{L}$ suspension. More detailed information was reported in our previous publications [8, 15].

\subsection{Plant Growth}

The seeds of Glycine max (L.) Merr. var. 'Tohya' were purchased from Johnny's Selected Seeds (Fairfield, MN). The seeds were sterilized with $2.7 \%$ hypochlorite bleach for $10 \mathrm{~min}$, washed with DI water three times and then germinated in a potting soil mix (Miracle Gro®, the Scotts Company, Marysville, OH). After 4 days of germination, the seedlings were transferred to $50 \mathrm{~mL}$ centrifuge tubes containing full strength Hoagland solution. The plant seedlings were cultivated at room temperature under a 16/8 h light cycle for 14 days before any treatment and the light density at the leaf level was about $250 \mu \mathrm{mol} \mathrm{m} \mathrm{s}^{-1}$. The seedlings age was chosen because seedlings at this age are physiologically relevant to the effects of the selected pharmaceutical doses $[16,17]$.

\subsection{Pre-treatment of Samples with Pharmaceutical Agents}

The pharmaceutical agents were mixed with one-fifth strength Hoagland solution to achieve the following concentrations: DNP: $50 \mu \mathrm{M}$; CHX: $20 \mu \mathrm{M}$; $\mathrm{LaCl}_{3}: 1 \mathrm{mM}$, and $\mathrm{GdCl}_{3} 100 \mu \mathrm{M}$. The $\mathrm{pH}$ of the individual solutions was adjusted to 6.0. For pre-treatment, healthy seedlings were exposed to solutions with the individual blocker compounds at the listed concentration for $12 \mathrm{~h}$.

\subsection{Uptake Experiment and Plant Harvest}

Immediately after the pre-treatment, the seedlings were washed five times with DI water and were transferred to 50 $\mathrm{mL}$ polypropylene centrifuge tubes containing $50 \mathrm{~mL}$ solutions of $10 \mathrm{mg} / \mathrm{L}$ of $\mathrm{CeO}_{2} \mathrm{NPs}$ or same concentration of $\mathrm{Ce}^{3+}$ in tap water. Altogether, 33 seedlings were grown in 11 different treatments: one negative control (no pretreatment and no Ce exposure); two positive controls (no pretreatment but exposed to either $\mathrm{CeO}_{2} \mathrm{NPs}$ or $\mathrm{Ce}^{3+}$ ) and eight treatments (pretreatment with one of the four pharmaceuticals and exposed to either $\mathrm{CeO}_{2} \mathrm{NPs}$ or Ce ions). The seedlings were harvested 5 days after treatment. During the uptake experiment, the transpired water was replenished with tap water. At termination, the roots were washed with $5.0 \mathrm{mM} \mathrm{CaCl}_{2}$ solution five times and then with DI water three times. Previous studies indicated that the $\mathrm{CaCl}_{2}$ solution is highly effective to remove $\mathrm{CeO}_{2} \mathrm{NPs}$ deposited on root surface[15]. After the roots were dried with a paper towel, the seedlings were separated into roots and shoots then dried in an oven at $75{ }^{\circ} \mathrm{C}$ for 48 hours and acid digested following published protocols [15, 17].

\subsection{Statistical Analysis}

Statistical analysis on the cerium concentration in plant tissues was performed using the Minitab 18 Statistical Software (Minitab Inc., State College, PA, USA). The comparison between mean values of different treatments was carried out using One-Way ANOVA, followed by Tukey's test at significance level 5\% $(p<0.05)$. 


\section{Results}

\subsection{Growth Analysis}

Pre-treatment of plant seedlings with selected pharmaceuticals had no impact on plant dry biomass, but the water transpiration of soybean seedlings was significantly reduced by $\mathrm{CHX}$. Sequential exposure to DNP and $\mathrm{CeO}_{2} \mathrm{NPs}_{\mathrm{s}}$ also significantly decreased the water transpiration, However, plants sequentially exposed to DNP and $\mathrm{Ce}^{3+}$ had statistically comparable transpiration rate to the $\mathrm{Ce}^{3+}$ controls, suggesting the different impact of $\mathrm{CeO}_{2} \mathrm{NPs}$ and $\mathrm{Ce}^{3+}$ on plants.

\subsection{Ce contents in Soybean Tissues Treated with $\mathrm{CeO}_{2} \mathrm{NPs}$}

Concentrations of $\mathrm{Ce}$ in soybean root and shoot tissues after different pretreatments and exposure to $10 \mathrm{mg} / \mathrm{L} \mathrm{of}$ $\mathrm{CeO}_{2} \mathrm{NPs}$ are shown in Figure 1. Ce concentration in soybean roots pretreated with $\mathrm{La}^{3+}$ was unaffected. Surprisingly, pretreatment with $\mathrm{Gd}^{3+}$ led to a $42 \%$ increase in Ce concentration in soybean roots. Ce concentration in soybean roots pretreated with DNP also increased significantly by $36 \%$ compared to the plants exposed to the same concentration of $\mathrm{CeO}_{2} \mathrm{NPs}$ without pretreatment. In contrast, pretreatment with CHX significantly reduced the Ce concentration in soybean roots by $35 \%$.

The Ce concentration in soybean shoots was also affected by the pretreatments. Only DNP significantly increased Ce concentration in soybean shoots by $21 \%$, while CHX caused a $17 \%$ decrease of Ce concentration in soybean shoots compared to the controls. Ce concentration in soybean shoots pretreated with $\mathrm{Gd}^{3+}$ was comparable to the controls even though the Ce concentration in $\mathrm{Gd}^{3+}$ pretreated soybean roots was markedly higher. Notably, the Ce concentration in soybean shoot was drastically reduced by $51 \%$ due to pretreat with $\mathrm{La}^{3+}$, even though $\mathrm{La}^{3+}$ did not affect Ce concentration in soybean roots.

\subsection{Ce Contents in Soybean Tissues Treated with Ce lons}

Similar to $\mathrm{CeO}_{2} \mathrm{NPs}$ treated plants, the Ce concentration in $\mathrm{Ce}^{3+}$ treated soybean roots and shoots was significantly elevated $(\mathrm{p}<0.05)$ by DNP pretreatment. In root tissues treated with CHX, the concentration of Ce was reduced by $67 \%$ $(\mathrm{p}<0.05)$. However, the Ce concentration in soybean shoots was unaffected. In comparison to the results observed for $\mathrm{CeO}_{2} \mathrm{NPs}, \mathrm{La}^{3+}$ and $\mathrm{Gd}^{3+}$ displayed different impacts on $\mathrm{Ce}$ ion uptake. Neither blockers affected the Ce concentration in soybean roots, however, $\mathrm{Gd}^{3+}$ was able to completely block the transport of Ce from root to shoot while $\mathrm{La}^{3+}$ only led to insignificant reduction of $\mathrm{Ce}$ in soybean shoots. Detailed results of different ion blockers or metalolic inhibitors on soybean uptake of Ce ions are shown in Figure 2.

\section{Discussion}

The generally different impact of chosen blockers and inhibitors, except for DNP, on the uptake and transport of $\mathrm{CeO}_{2} \mathrm{NPs}$ and $\mathrm{Ce}^{3+}$ suggest that they follow different routes and mechanisms of plant root uptake and in-planta transport. DNP reduces the production of high-energy phosphate bonds in mitochondria and inhibits the formation of Adenosine triphosphate (ATP), an energy source for regulating the gate action and transport of divalent ions through the calciumpermeable channels [18]. Shortage of metabolic energy due to DNP pretreatment might have affected the regulation of channel opening and allowed more $\mathrm{Ce}$ to pass through the channels. The significant increase of $\mathrm{Ce}$ in plant tissues exposed to both $\mathrm{CeO}_{2} \mathrm{NPs}$ and $\mathrm{Ce}$ ion following DNP pretreatment suggests that calcium-permeable channels are generally not available for Ce uptake, but they may potentially be used for transporting both Ce when their regulation is compromised. Because $\mathrm{CeO}_{2} \mathrm{NPs}$ dissolute in plant rhizosphere and release $\mathrm{Ce}^{3+}$ [15], and DNP pretreatment also lead to higher $\mathrm{Ce}$ in $\mathrm{Ce}^{3+}$ treated soybeans, it is possible that the elevated $\mathrm{Ce}$ in $\mathrm{CeO}_{2} \mathrm{NPs}$ treated soybeans was due to greater transport of dissolved $\mathrm{Ce}^{3+}$, not intact $\mathrm{CeO}_{2} \mathrm{NPs}$.

Trivalent lanthanide elements gadolinium $\left(\mathrm{Gd}^{3+}\right)$ and lanthanum $\left(\mathrm{La}^{3+}\right)$ are both close to the divalent calcium ion $\left(\mathrm{Ca}^{2+}\right)$ in size (radius of $\mathrm{Gd}^{3+} 1.05-1.11 \AA$ and $\mathrm{Ca}^{2+} 1.00-1.06 \AA$ ), electronegativity, bonding, and coordination [19]. Their inhibitory behavior for $\mathrm{Ca}^{2+}$ transport through calcium channels arises from their competition with $\mathrm{Ca}^{2+}$ for binding sites [20]. Interestingly, these two blockers displayed highly different impacts of on the uptake of $\mathrm{CeO}_{2} \mathrm{NPs}_{\mathrm{s}}$ and $\mathrm{Ce}^{3+}$. While the pretreatment with $\mathrm{La}^{3+}$ had little impact on $\mathrm{CeO}_{2} \mathrm{NPs}$ uptake and transport, $\mathrm{Gd}^{3+}$ significantly increased Ce concentration in soybean roots exposed to $\mathrm{CeO}_{2} \mathrm{NPs}$. Their impacts on plant uptake of $\mathrm{Ce}^{3+}$ also differed. Even though neither blockers significantly altered $\mathrm{Ce}$ concentration in $\mathrm{Ce}^{3+}$ exposed soybean roots, the transport of $\mathrm{Ce}^{3+}$ to soybean shoots was completely shut down by $\mathrm{Gd}^{3+}$. The results suggest that the in-planta transport for $\mathrm{CeO}_{2} \mathrm{NPs}$ and $\mathrm{Ce}^{3+}$ are substantially different. 
Pretreatment with $\mathrm{CHX}$ reduced $\mathrm{Ce}$ in plant root tissues for both $\mathrm{CeO}_{2} \mathrm{NPs}$ and $\mathrm{Ce}^{3+}$ treated plant roots due to the decreased water uptake. The lower water transpiration caused by CHX pretreatment, together with the reduced Ce concentration in plant roots, suggest that both $\mathrm{CeO}_{2} \mathrm{NPs}_{\text {and }} \mathrm{Ce}^{3+}$ can be taken up by plant roots and transported to plant shoots with the transpired water and aquaporins may play a role in their uptake.

Overall, the results confirmed the differential uptake and transport mechanisms of $\mathrm{CeO}_{2} \mathrm{NPs}$ and $\mathrm{Ce}^{3+}$. The results suggested that aquaporins in plant roots may play a role in the uptake of $\mathrm{CeO}_{2} \mathrm{NPs}$ through their control on plant water uptake. Disturbance on the regulation of transporter proteins in plant roots could affect the plant uptake of $\mathrm{CeO}_{2} \mathrm{NPs}$ and their channels can be potentially available for $\mathrm{CeO}_{2} \mathrm{NPs}$ transport when their regulation is compromised. Alternatively, due to the widespread presence of pharmaceutical compounds, it is imperative to under how these compounds could potentially alter the plant uptake of transport of co-existing ENPs.

\section{References}

[1] Tourinho, P.S., et al., Metal-based nanoparticles in soil: Fate, behavior, and effects on soil invertebrates. Environmental Toxicology and Chemistry, 2012. Vol.31, no. 8, pp. 1679-1692.

[2] Sharifan, H. and X. Ma, Potential Photochemical Interactions of UV Filter Molecules with Multi-chlorinated Structure of Prymnesins in Harmful Algal Bloom Events. Mini Review in Organic Chemistry, 2017.

[3] Benn, T.M. and P. Westerhoff, Nanoparticle silver released into water from commercially available sock fabrics. Environmental science \& technology, 2008. Vol. 42, no. 11, pp. 4133-4139.

[4] Rafique, M., et al., Dependence of the structural optical and thermo-physical properties of gold nano-particles synthesized by laser ablation method on the nature of laser. Optik - International Journal for Light and Electron Optics, 2017. Vol. 134, pp. 140-148.

[5] Tripathi, D.K., et al., An overview on manufactured nanoparticles in plants: Uptake, translocation, accumulation and phytotoxicity. Plant Physiology and Biochemistry, 2017. Vol. 110, pp. 2-12.

[6] Servin, A.D., et al., Exposure of agricultural crops to nanoparticle CeO2 in biochar-amended soil. Plant Physiology and Biochemistry, 2017. Vol. 110, pp. 147-157.

[7] Schymura, S., et al., Elucidating the Role of Dissolution in CeO2 Nanoparticle Plant Uptake by Smart Radiolabeling. Angewandte Chemie International Edition, 2017.

[8] Zhang, W., et al., Uptake and accumulation of bulk and nanosized cerium oxide particles and ionic cerium by radish (Raphanus sativus L.). Journal of agricultural and food chemistry, 2015. Vol. 63, no. 2, pp. 382-390.

[9] Zuverza-Mena, N., et al., Exposure of engineered nanomaterials to plants: Insights into the physiological and biochemical responses-A review. Plant Physiology and Biochemistry, 2017. Vol. 110, pp. 236-264.

[10] Anjum, N.A., et al., Transport phenomena of nanoparticles in plants and animals/humans. Environmental Research, 2016. Vol. 151, pp. 233-243.

[11] Zhao, L., et al., Effect of surface coating and organic matter on the uptake of CeO2 NPs by corn plants grown in soil: Insight into the uptake mechanism. Journal of Hazardous Materials, 2012. Vol. 225, pp. 131-138.

[12] Li, L.-Z., et al., Characteristics of cadmium uptake and membrane transport in roots of intact wheat (Triticum aestivum L.) seedlings. Environmental Pollution, 2017. Vol. 221, pp. 351-358.

[13] Li, L.Z., et al., Pathways of root uptake and membrane transport of Cd2+ in the zinc/cadmium hyperaccumulating plant Sedum plumbizincicola. Environmental Toxicology and Chemistry, 2016.

[14] Osborne, S.A., et al., Has the sensitivity of soybean cultivars to ozone pollution increased with time? An analysis of published dose-response data. Global change biology, 2016. Vol. 22, no. 9, pp. 3097-3111.

[15] Zhang, W., et al., Elucidating the mechanisms for plant uptake and in-planta speciation of cerium in radish (Raphanus sativus L.) treated with cerium oxide nanoparticles. Journal of Environmental Chemical Engineering, 2017. Vol. 5, no. 1, pp. 572-577.

[16] Loughman, B.C., METABOLIC PROCESSES IN ROOTS RELATED TO ABSORPTION AND TRANSPORT OF PHOSPHATE A2 - SUNDERLAND, N, in Botany. 1976, Pergamon. pp. 423-431. 
[17] Sharifan, H., Commentary on "Characteristics of cadmium uptake and membrane transport in roots of intact wheat (Triticum aestivum L.) seedlings" reported by Lian-Zhen Li, Chen Tu, Willie JGM Peijnenburg, Yong-Ming Luo. Environmental Pollution, 2017.

[18] Cao, Z., et al., Physiological effects of cerium oxide nanoparticles on the photosynthesis and water use efficiency of soybean (Glycine max (L.) Merr.). Environmental Science: Nano, 2017. Vol. 4, no. 5, pp. 10861094.

[19] Rahwan, R.G., D.T. Witiak, and W.W. Muir, Chapter 23. Calcium Antagonists. Annual Reports in Medicinal Chemistry, 1981. Vol. 16: pp. 257-268.

[20] Bouron, A., K. Kiselyov, and J. Oberwinkler, Permeation, regulation and control of expression of TRP channels by trace metal ions. Pflügers Archiv - European Journal of Physiology, 2015. Vol. 467, no. 6: pp. 1143-1164.
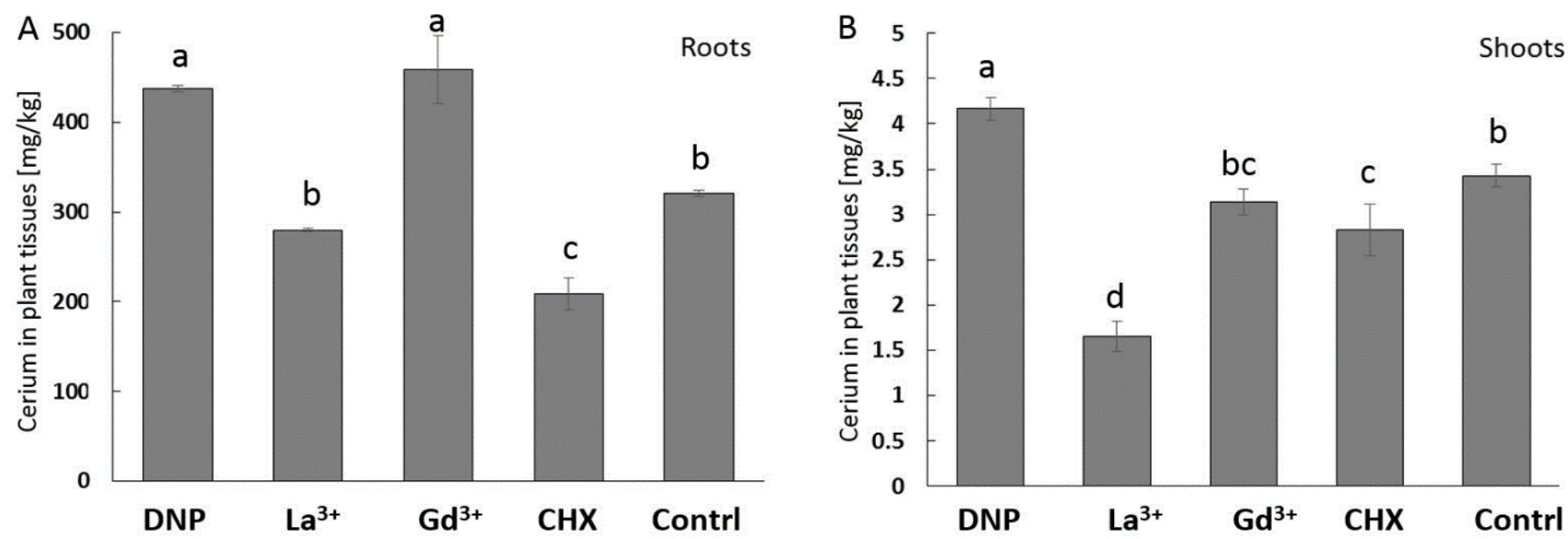

Fig. 1: Effects of various inhibitors on the concentration of Ce in soybean tissues exposed to $10 \mathrm{ppm} \mathrm{CeO}_{2} \mathrm{NPs}_{\mathrm{N}}$ suspension (A) in roots and (B) in shoots. The reported Ce concentrations are means \pm S.D. $(n=3)$. The letters above the bars indicate the statistical grouping of different treatments $(p<0.05)$.
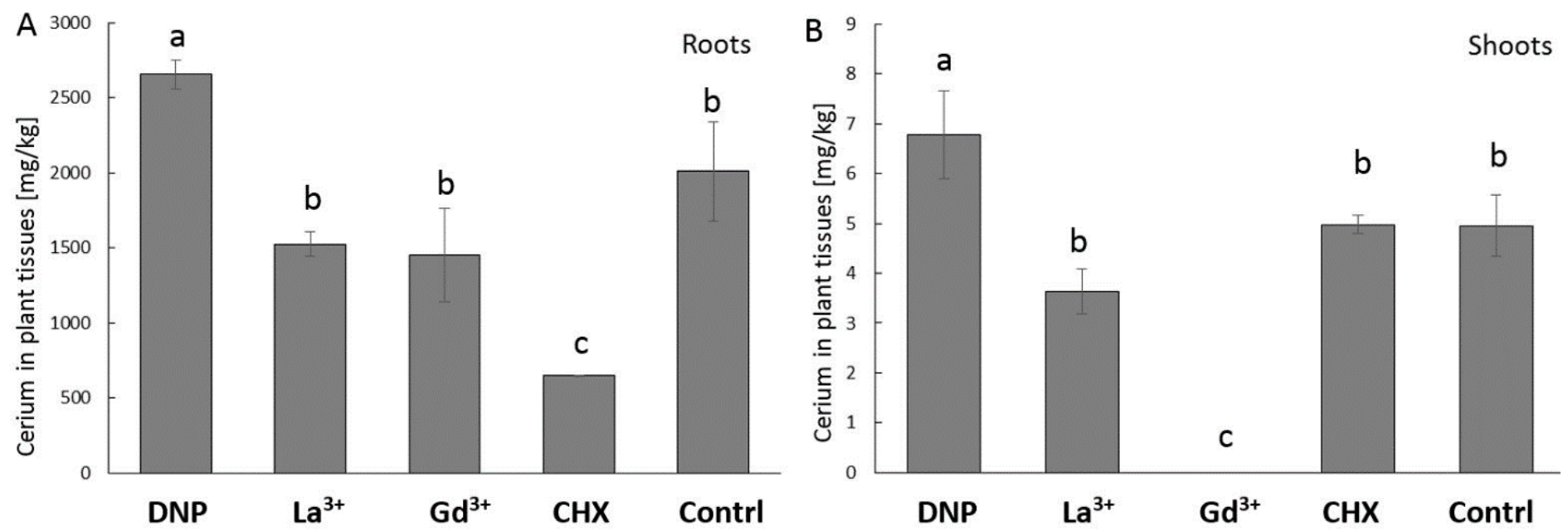
Fig. 2: Effects of various inhibitors on the uptake of Ce ion (A) in roots and (B) in roots of soybeans after exposure to 10 ppm Ce ions. The reported values are means \pm S.D. $(n=3)$. Letters above bars represent significant differences $(p<0.05)$. 